\title{
A Super-Replication Theorem in Kabanov's Model of Transaction Costs
}

\author{
Luciano Campi* Walter Schachermayer ${ }^{\dagger \ddagger}$
}

June 26, 2006

\begin{abstract}
We prove a general version of the super-replication theorem, which applies to Kabanov's model of foreign exchange markets under proportional transaction costs. The market is described by a matrix-valued càdlàg bid-ask process $\left(\Pi_{t}\right)_{t \in[0, T]}$ evolving in continuous time.

We propose a new definition of admissible portfolio processes as predictable (not necessarily right or left continuous) processes of finite variation related to the bid-ask process by economically meaningful relations. Under the assumption of existence of a Strictly Consistent Price System (SCPS), we prove a closure property for the set of attainable vector-valued contingent claims. We then obtain the super-replication theorem as a consequence of that property, thus generalizing to possibly discontinuous bid-ask processes analogous results obtained by Kabanov [11], Kabanov and Last [12] and Kabanov and Stricker [15]. Rásonyi's counter-example [16] served as an important motivation for our approach.
\end{abstract}

Keywords: proportional transaction costs, foreign exchange markets, uniform efficient friction, super-replication theorem.

JEL classification: G10, G11, G13.

AMS Classification (2000): Primary 91B28; Secondary 60H30, 60G44.

\section{Introduction}

The term "super-replication theorem" or "hedging theorem" denotes a class of results describing the set of initial endowments allowing an investor to hedge (or super-replicate) a given contingent claim by pursuing some self-financing trading strategy.

*Vienna University of Technology, Financial and Actuarial Mathematics, Wiedner Hauptstraße 8-10/1051, A-1040 Vienna, Austria. E-mail: lucianocampi@yahoo.it.

${ }^{\dagger}$ Vienna University of Technology, Financial and Actuarial Mathematics, Wiedner Hauptstraße 8-10/1051, A-1040 Vienna, Austria and Université Paris Dauphine, Place du Maréchal de Lattre de Tassigny, F-75775 Paris Cedex 16, France. E-mail: wschach@fam.tuwien.ac.at.

${ }^{\ddagger}$ We gratefully acknowledge financial support for both authors from the Austria Science Fund (FWF) under the Wittgenstein-Preis program Z36 and grant P15889 as well as from the Research Training Network HPRN-CT-2002-00281. Thanks also go to Yuri Kabanov for numerous suggestions and comments on a previous version of this paper and to two anonymous referees. 
In a frictionless financial market, this set coincides - under suitable regularity assumptions - with the set of all initial investments greater than every expectation of the contingent claim calculated with respect to all equivalent (local) martingale measures (see e.g. [9], [1] and $[6])$.

The absence of friction assumption is clearly a strong idealization of the real world and much effort has been devoted to the super-hedging problem in the presence of transaction costs.

In a financial market with proportional transaction costs as modelled by Kabanov [11], establishing a super-replication theorem is a somewhat delicate task. In this setting, a contingent claim is modelled by an $\mathbb{R}^{d}$-valued random variable representing e.g. positions in several currencies, so that the initial endowments are vector-valued, too.

In a discrete time setting, a complete characterization of the set of initial investments necessary to super-hedge a given contingent claim has been given in [5], [13], [14] and [17].

In a continuous time framework, the problem is more delicate. A subtle point is the definition of a "good" notion of admissible trading strategy. A first solution has been given by Kabanov [11], by assuming constant transaction costs and continuous price processes with respect to a given numéraire. His results rely heavily on the underlying $L^{2}$ structure. An improvement has been made by Kabanov and Last [12], by dropping the integrability condition that characterizes admissible strategies in [11] and replacing it with the more natural boundedness from below property, that had turned out to be useful in the frictionless case (see e.g. [6]). Also in [12], the authors assume that the transaction costs are constant over time and that the numéraire has continuous price dynamics.

A further step forward is the paper [15] by Kabanov and Stricker, where the solvency cones $\widehat{K}_{t}(\omega)$ induced by the financial market are now modelled as time-dependent and random. In [15], they are assumed to be generated by a countable family of continuous processes.

In all those papers, the authors work under the efficient friction assumption, which roughly means that the transaction costs are strictly positive, thus excluding the frictionless case from their setting. Moreover, an admissible self-financing trading strategy is defined as an adapted, vector-valued, càdlàg process of finite variation whose increments lie in the solvency cones and its terminal value is bounded from below by a constant with respect to the order induced by the cone $\widehat{K}_{T}$.

In the present paper we extend these results to bid-ask processes which are not necessarily continuous and prove a general super-replication theorem in this framework. There is an enlightening counter-example, due to M. Rásonyi [16], which - at first glance - seems to make such an effort bound to fail: roughly speaking, in this example, which uses a bid-ask process with jumps, there is a sequence of càdlàg portfolio processes $\left(\widehat{V}_{t}^{n}\right)_{t \in[0, T]}$, where $\widehat{V}^{n}$ is defined via a trade at time $t_{n}=1-n^{-1}$. If one tries to pass to a limit of this sequence - which is always the crucial step in the proof of a super-replication theorem - one faces a difficulty: the limiting process $\widehat{V}^{0}$ would naturally be defined by a trading which is done at time $t=1-$, i.e., "immediately before time 1", which should be distinguishable from trading at time $t=1$. But such a distinction is not possible in the framework of càdlàg portfolio processes $\left(\widehat{V}_{t}\right)_{t \in[0, T]}$ as used in the previous literature. We also note that such a 
distinction is not necessary in the case of continuous bid-ask processes, but if one allows for jumps of these processes, this issue becomes unavoidable, as convincingly pointed out by Rásonyi's example.

Our idea to remedy this problem is very simple: we model the portfolio processes $\left(\widehat{V}_{t}\right)_{t \in[0, T]}$, where the $\mathbb{R}^{d}$-valued random variable $\widehat{V}_{t}$ describes the portfolio of $d$ assets held at time $t$, as predictable processes. Recall that, for a predictable process $\left(\widehat{V}_{t}\right)_{t \in[0, T]}$ and a predictable stopping time $\tau$ (e.g., a deterministic time such as $\tau \equiv 1$ ) it is perfectly possible that the values $\widehat{V}_{\tau-}=\lim _{t \uparrow \tau} \widehat{V}_{t}$ and $\widehat{V}_{\tau+}=\lim _{t \downarrow \tau} \widehat{V}_{t}$ both are different from $\widehat{V}_{\tau}$, so that they allow for modelling a jump $\Delta \widehat{V}_{\tau}=\widehat{V}_{\tau}-\widehat{V}_{\tau-}$ "immediately before time $\tau$ " as well as a jump $\Delta_{+} \widehat{V}_{\tau}=\widehat{V}_{\tau+}-\widehat{V}_{\tau}$ "at time $\tau$ ".

In fact, we believe that it is very natural to model portfolio processes in a predictable way (at least in the present context of bid-ask processes with jumps). Recall that in the classical frictionless setting, where the financial market is modelled by an $\mathbb{R}^{d}$-valued semimartingale $\left(S_{t}\right)_{t \in[0, T]}$ (see, e.g., [6]), the trading strategies $\left(H_{t}\right)_{t \in[0, T]}$, where the $\mathbb{R}^{d}$-valued random variable $H_{t}$ describes the portfolio of $d$ assets held at time $t$, are also modelled as predictable processes so that the stochastic integral $(H \cdot S)_{T}=\int_{0}^{T} H_{t} d S_{t}$ makes sense. Turning again to the case of financial markets with transaction costs, we also observe that - in nuce - already in discrete time the necessity occurs of modelling the possibility of a trade "before the change of prices" as well as "after the change of prices". Indeed, think of a one-period bid-ask process $\left(\Pi_{t}\right)_{t=0}^{1}$ as in [17]: there is one change of prices but two possibilities of trade, namely at time $t=0$, i.e. before the change of prices, and $t=1$, i.e., after the change of prices.

In Section 2 below we develop the notion of admissible predictable portfolio processes. We introduce the notion of "Strictly Consistent Price System" (SCPS), which is the continuous time version of a well-established concept known to be useful in the present context (compare with [15], Section 3.1). We also compare our notion of admissibility with that which appeared in the paper [12] in the case of constant transaction costs.

In Section 3 we prove the main result of this paper: if there exists an SCPS, the set of attainable contingent claims is Fatou-closed with respect to the order induced by $\widehat{K}_{T}$. Roughly speaking, this means that our framework is designed in such a way, that it is indeed possible to pass from a sequence $\left(\widehat{V}_{t}^{n}\right)_{t \in[0, T]}$ of portfolio processes (that are uniformly bounded from below in a sense to be made precise below) to a limiting portfolio process $\left(\widehat{V}_{t}\right)_{t \in[0, T]}$. A central tool, which may also be of some independent interest, is Proposition13: it is a parametrized version of (a strengthening of) Helly's theorem on pointwise convergence of sequences $\left(f_{n}\right)_{n=1}^{\infty}$ of functions on $[0, T]$ of uniformly bounded variation.

Finally, in Section 4 we apply this result to deduce the super-replication theorem. This final step is rather standard.

\section{The Model: Terminology and Definitions}

We follow the terminology established in [17]. Due to the fact that we are working in continuous time, some additional concepts will be necessary. 
Let $\left(\Omega,\left(\mathcal{F}_{t}\right)_{t \in[0, T]}, \mathbf{P}\right)$ be a filtered probability space satisfying the usual conditions (i.e. it is right-continuous and $\mathbf{P}$-saturated). We assume without loss of generality that $\mathcal{F}_{0}$ is trivial, i.e., consists only of the null sets and their complements. We recall the definition of a bid-ask matrix and a bid-ask process:

Definition $1 A d \times d$ matrix $\Pi=\left(\pi^{i j}\right)_{1 \leq i, j \leq d}$ is called a bid-ask matrix if:

1. $\pi^{i j}>0$ for every $1 \leq i, j \leq d$;

2. $\pi^{i i}=1$ for every $1 \leq i \leq d$;

3. $\pi^{i j} \leq \pi^{i k} \pi^{k j}$, for every $1 \leq i, j, k \leq d$.

An adapted, càdlàg process $\left(\Pi_{t}\right)_{t \in[0, T]}$ taking values in the set of bid-ask matrices will be called a bid-ask process.

Given a bid-ask matrix $\Pi$, we can define the following objects, that will play a major role in the definition of portfolio processes.

- the solvency cone $\widehat{K}(\Pi)$, which is the convex cone in $\mathbb{R}^{d}$ spanned by the unit vectors $e^{i}, 1 \leq i \leq d$, and the vectors $\pi^{i j} e^{i}-e^{j}, 1 \leq i, j \leq d$;

- the cone of portfolios available at price zero $-\widehat{K}(\Pi)=\left\{x \in \mathbb{R}^{d}:-x \in \widehat{K}(\Pi)\right\}$;

- the polar of the cone $-\widehat{K}(\Pi)$, denoted by $\widehat{K}^{*}(\Pi)$ and given by

$$
\begin{aligned}
\widehat{K}^{*}(\Pi) & =\left\{w \in \mathbb{R}^{d}:\langle v, w\rangle \leq 0, \forall v \in-\widehat{K}(\Pi)\right\} \\
& =\left\{w \in \mathbb{R}^{d}:\langle v, w\rangle \geq 0, \forall v \in \widehat{K}(\Pi)\right\} .
\end{aligned}
$$

We call the elements $w \in \widehat{K}^{*}(\Pi)$ (resp. in the relative interior of $\widehat{K}^{*}(\Pi)$ ) price systems consistent (resp. strictly consistent) with the bid-ask matrix $\Pi$.

For the financial interpretation and some comments about the previous definitions, we refer to [17].

From now on, the bid-ask process $\left(\Pi_{t}\right)_{t \in[0, T]}$ will be fixed once and for all, so that we can drop it from the notation, by writing e.g. $\widehat{K}_{\tau}$ and $\widehat{K}_{\tau-}$ instead of, respectively, $\widehat{K}\left(\Pi_{\tau}\right)$ and $\widehat{K}\left(\Pi_{\tau-}\right)$, for a stopping time $\tau$. Observe that, since $\left(\Pi_{t}\right)_{t \in[0, T]}$ is right-continuous, we have that $\widehat{K}_{\tau+}=\widehat{K}_{\tau}$ a.s. for every stopping time $\tau$.

The cones $\left(\widehat{K}_{t}\right)_{t \in[0, T]}$, induce a natural order among $\mathbb{R}^{d}$-valued random variables. Let $\tau$ be a stopping time and let $U, V$ be two $\mathcal{F}_{\tau}$-measurable random variables. We will write $U \succeq_{\tau} V$, if $U-V \in L^{0}\left(\widehat{K}_{\tau}, \mathcal{F}_{\tau}\right)$, the cone of all $\widehat{K}_{\tau}$-valued $\mathcal{F}_{\tau}$-measurable random variables.

In order to avoid the necessity of developing special notation for possible trading at the final date $T$, we will work, without loss of generality, under the following technical assumption concerning the filtration $\left(\mathcal{F}_{t}\right)_{t \in[0, T]}$ and the bid-ask process $\left(\Pi_{t}\right)_{t \in[0, T]}$ :

Assumption $2 \mathcal{F}_{T-}=\mathcal{F}_{T}$ and $\Pi_{T-}=\Pi_{T}$ a.s. 
We will explain in the last section, Remark 16, why this assumption does not restrict the generality of the model. We now give the definitions of consistent and strictly consistent price processes.

Definition 3 An adapted $\mathbb{R}_{+}^{d} \backslash\{0\}$-valued process $Z=\left(Z_{t}\right)_{t \in[0, T]}$ is called a consistent price process for the bid-ask process $\left(\Pi_{t}\right)_{t \in[0, T]}$ if:

- $Z$ is a càdlàg $\mathbf{P}$-martingale;

- for every $t \in[0, T], Z_{t} \in \widehat{K}_{t}^{*}$ a.s.

Moreover, if a consistent price process $Z$ satisfies the following additional condition:

- for every $[0, T] \cup\{\infty\}$-valued stopping time $\tau, Z_{\tau} \in \operatorname{int} \widehat{K}_{\tau}^{*}$ a.s. on $\{\tau<\infty\}$, and for every predictable $[0, T] \cup\{\infty\}$-valued stopping time $\sigma, Z_{\sigma-} \in \operatorname{int} \widehat{K}_{\sigma-}^{*}$ a.s. on $\{\sigma<\infty\}$,

then $Z$ will be called a strictly consistent price process. The set of all (strictly) consistent price processes will be denoted by $\mathcal{Z}\left(\mathcal{Z}^{s}\right)$.

Remark 4 For a consistent price process $Z$ and for a random time $\tau, Z_{\tau} \in \widehat{K}_{\tau}^{*}$ a.s. Indeed, for each random time $\tau$, there exists a sequence $\tau_{n}$ of random times taking finitely many values and such that $\tau_{n} \downarrow \tau$ a.s.. Then, since $Z$ is assumed right-continuous, by letting $n$ tend to infinity, we have $Z_{\tau} \in \widehat{K}_{\tau}^{*}$ a.s.. Similarly, if $\sigma$ is a predictable $[0, T] \cup\{\infty\}$-valued stopping time, then $Z_{\sigma-} \in \widehat{K}_{\sigma-}^{*}$ a.s. on $\{\sigma<\infty\}$.

We now introduce the standing assumption of the paper, stating that there exists a strictly consistent price process $Z^{s}$ for the financial market described by the bid-ask process $\left(\Pi_{t}\right)_{t \in[0, T]}$.

Assumption 5 Existence of a Strictly Consistent Price System (SCPS): $\mathcal{Z}^{s} \neq \emptyset$.

Remark 6 Observe that SCPS implies a property that in the transaction costs literature (e.g., see [15]) is usually called Efficient Friction (EF):

$$
\operatorname{int} \widehat{K}_{t}^{*} \neq \emptyset \quad \text { a.s., for every } t \in[0, T] .
$$

We will say that a process $X$ is of finite variation if almost each of its paths has finite variation. We do not assume that $X$ is necessarily right or left-continuous as in most textbooks on stochastic calculus (e.g. [8], [10]). We will denote by $\operatorname{Var}_{t}(X)$ the total variation of $X$ on $[0, t]$, i.e.

$$
\operatorname{Var}_{t}(X)=\sup _{0 \leq t_{0} \leq \ldots \leq t_{n} \leq t} \sum_{i=0}^{n-1}\left\|X_{t_{i+1}}-X_{t_{i}}\right\|_{d}, \quad t \in[0, T] .
$$

Here $\|\cdot\|_{d}$ denotes the Euclidean norm on $\mathbb{R}^{d}$. 
For a given process of finite variation $X$, we set

$$
X_{t}^{c}:=X_{t}-\sum_{s<t} \Delta_{+} X_{s}-\sum_{s \leq t} \Delta X_{s}
$$

where $\Delta_{+} X_{t}:=X_{t+}-X_{t}$ and $\Delta X_{t}:=X_{t}-X_{t-}, t \in[0, T]$. By convention, $\Delta X_{0}=\Delta_{+} X_{T}=$ 0 . If moreover $X$ has continuous paths, then we denote by $\left(\dot{X}_{t}\right)_{t \in[0, T]}$ its Radon-Nikodym derivative with respect to the scalar increasing function $\left(\operatorname{Var}_{t}(X)\right)_{t \in[0, T]}$. Note that $\dot{X}_{t}(\omega)$ is well-defined and takes its values in the unit sphere of $\mathbb{R}^{d}$ a.e. with respect to the product measure $d \mathbf{P} \otimes d \operatorname{Var}_{t}(X)$ defined on $\Omega \times[0, T]$.

We now introduce the central concept of this paper.

Definition 7 Suppose that $\left(\Pi_{t}\right)_{t \in[0, T]}$ is a bid-ask process such that SCPS holds true. An $\mathbb{R}^{d}$-valued process $\widehat{V}=\left(\widehat{V}_{t}\right)_{t \in[0, T]}$, is called a self-financing portfolio process for the bid-ask process $\left(\Pi_{t}\right)_{t \in[0, T]}$ if it satisfies the following properties:

(i) it is predictable and a.e. path has finite variation;

(ii) for every pair of stopping times $0 \leq \sigma \leq \tau \leq T$, we have

$$
\widehat{V}_{\tau}-\widehat{V}_{\sigma} \in-\widehat{\mathcal{K}}_{\sigma, \tau} \quad \text { a.s. }
$$

where

$$
\widehat{\mathcal{K}}_{\sigma, \tau}(\omega):=\overline{\operatorname{conv}}\left(\bigcup_{\sigma(\omega) \leq u<\tau(\omega)} \widehat{K}_{u}(\omega), 0\right),
$$

the bar denoting closure in $\mathbb{R}^{d}$.

Furthermore, a self-financing portfolio process $\widehat{V}$ will be called admissible if it satisfies the following additional property:

(iii) there is a threshold, i.e., a constant $a>0$ such that $\widehat{V}_{T} \succeq_{T}-a \mathbf{1}$ and $Z_{\tau}^{s} \widehat{V}_{\tau} \geq-a Z_{\tau}^{s} \mathbf{1}$ a.s. for all $[0, T]$-valued stopping times $\tau$ and for every strictly consistent price process $Z^{s} \in \mathcal{Z}^{s}$.

We denote by $\widehat{\mathcal{V}}_{\text {adm }}$ the set of all such portfolio processes and set

$$
\widehat{\mathcal{V}}_{a d m}^{x}:=\left\{\widehat{V} \in \widehat{\mathcal{V}}_{a d m}: \widehat{V}_{0}=x\right\},
$$

for some initial endowment $x \in \mathbb{R}^{d}$.

Some remarks seem in order to motivate this definition. The assumption of predictability was discussed in the introduction and this seems to us the most basic feature of the above concept. The condition of a.s. finite variation is related to the efficient friction (EF) property: the economic rationale behind it is that a portfolio process having trajectories 
with infinite variation would be a very stupid investment strategy, as the transaction costs would add up to infinity for the trajectories with infinite variation. Therefore we may restrict to portfolio processes with a.e. trajectory of finite variation. We give a precise version of this economic intuition in Lemma 11 below.

Let us now discuss condition (ii): fixing stopping times $\sigma \leq \tau$, the change $\widehat{V}_{\tau}(\omega)-\widehat{V}_{\sigma}(\omega)$ of the portfolio should be a.s. in the closure of the sum of the cones $\left(-\widehat{K}_{u}(\omega)\right)_{u \in[\sigma(\omega), \tau(\omega))}$ of contingent claims available (at time $u$ ) at price zero, which makes sense economically. Another way of making sense of the economic idea that the portfolio process $\widehat{V}$ can only evolve according to the terms of trade modelled via the cones $\left(\widehat{K}_{t}\right)_{t \in[0, T]}$ is to argue infinitesimally and to require that $d \widehat{V}_{t}$ should lie in $-\widehat{K}_{t}$ a.s., for all $0 \leq t \leq T$, as well as $d \widehat{V}_{t-}$ in $-\widehat{K}_{t-}$. This approach was used in [3], [4], [11], [12] and [19] among others, and works well in the context of the continuous modelization of $\left(\widehat{K}_{t}\right)_{t \in[0, T]}$ and càdlàg processes $\left(\widehat{V}_{t}\right)_{t \in[0, T]}$. In our present modelling we have preferred not to use infinitesimals and to formulate things in finite terms as in (2) above. We shall show in Lemma 8, how to relate this definition to the infinitesimal intuition.

Finally, let us discuss the notion of admissibility: condition $\widehat{V}_{T} \succeq_{T}-a \mathbf{1}$ in (iii) is in the usual spirit of uniform boundedness from below; however, in contrast to the frictionless case (e.g. [18] or [20]), we only impose such a condition at the terminal date $T$. In order to rule out doubling strategies and similar schemes we use the family of strictly consistent pricing systems (which is non-empty by Assumption 5). Writing condition (iii) as $Z_{\tau}^{s} \widehat{V}_{\tau} \geq Z_{\tau}^{s}(-a \mathbf{1})$ we may interpret this inequality in the following way: given any stopping time $\tau$ and any strictly consistent system $Z_{\tau}^{s}$ of prices at time $\tau$, the value $Z_{\tau}^{s} \widehat{V}_{\tau}=\sum_{i=1}^{d}\left(Z_{\tau}^{s}\right)^{i} \widehat{V}_{\tau}^{i}$ of the portfolio under the price system $Z_{\tau}^{s}$ is greater than or equal to the value $Z_{\tau}^{s}(-a \mathbf{1})$ of the portfolio $-a \mathbf{1}$ which consists of a short position in each asset $i=1, \ldots, d$. We refer to Proposition 9 below for more on this topic, and in particular for a comparison between our notion of admissibility and the one used by Kabanov and his co-authors in [12] and [15].

In the following lemma, we link our definition of portfolio processes to that given by Kabanov and Stricker [15]. In particular, we prove that if $\widehat{V}$ is admissible in the sense of Definition 7 then every product $Z \widehat{V}$ (with $Z \in \mathcal{Z}$ ) is a true super-martingale.

Lemma 8 Let $\left(\Pi_{t}\right)_{t \in[0, T]}$ be a bid-ask process such that $S C P S$ holds true and let $\widehat{V}$ be a predictable process of finite variation (not necessarily right or left-continuous). The following two assertions are equivalent:

1. (i) for a.e. $\omega \in \Omega$ and for $d \operatorname{Var}_{t}\left(\widehat{V}^{c}\right)(\omega)$-a.e. $t \in[0, T], \dot{\widehat{V}}_{t}^{c}(\omega) \in-\widehat{K}_{t}(\omega)$;

(ii) for every stopping time $\tau, \Delta_{+} \widehat{V}_{\tau} \in-\widehat{K}_{\tau}$ a.s.,

(iii) for every predictable stopping time $\sigma, \Delta \widehat{V}_{\sigma} \in-\widehat{K}_{\sigma-}$ a.s.;

2. for every pair of stopping times $\sigma \leq \tau, \widehat{V}_{\tau}-\widehat{V}_{\sigma} \in-\widehat{\mathcal{K}}_{\sigma, \tau}$ a.s.

Moreover, if the conditions are verified we have that $Z \widehat{V}$ is a local super-martingale for every $Z \in \mathcal{Z}$. If $\widehat{V}$ is also admissible, then $Z^{s} \widehat{V}$ is a true super-martingale for every $Z^{s} \in \mathcal{Z}^{s}$. 
Proof. 1. $\Rightarrow 2$. Let $\sigma \leq \tau$ be two stopping times. Observe that

$$
\widehat{V}_{\tau}-\widehat{V}_{\sigma}=\widehat{V}_{\tau}^{c}-\widehat{V}_{\sigma}^{c}+\sum_{\sigma \leq u<\tau} \Delta_{+} \widehat{V}_{u}+\sum_{\sigma<u \leq \tau} \Delta \widehat{V}_{u}
$$

Since, $\Delta_{+} \widehat{V}_{\tau} \in-\widehat{K}_{\tau}$, for all stopping times $\tau$, and $\Delta \widehat{V}_{\sigma} \in-\widehat{K}_{\sigma-}$ for all predictable stopping times $\sigma$, we have $\sum_{\sigma \leq u<\tau} \Delta_{+} \widehat{V}_{u} \in-\widehat{\mathcal{K}}_{\sigma, \tau}$ and $\sum_{\sigma<u \leq \tau} \Delta \widehat{V}_{u} \in-\widehat{\mathcal{K}}_{\sigma, \tau}$. Moreover, property 1. clearly yields that

$$
\widehat{V}_{\tau}^{c}-\widehat{V}_{\sigma}^{c}=\int_{\sigma}^{\tau} \dot{V}_{u}^{c} d \operatorname{Var}_{u}\left(\widehat{V}^{c}\right) \in-\widehat{\mathcal{K}}_{\sigma, \tau}, \quad \text { a.s.. }
$$

$2 . \Rightarrow 1$. To show that, for every stopping time $\tau$, the jump from the right $\Delta \widehat{V}_{\tau+}$ belongs to $-\widehat{K}_{\tau}$ a.s., it suffices to approximate $\tau$ by a sequence $\tau_{n}$ of stopping times such that $\tau_{n} \downarrow \tau$, as $n \rightarrow \infty$, and use again the right-continuity of the bid-ask process $\left(\Pi_{t}\right)_{t \in[0, T]}$. For the jump from the left at any predictable stopping time $\sigma$, let us consider an announcing sequence $\sigma_{n}$ for $\sigma$. By 2 .

$$
\widehat{V}_{\sigma}-\widehat{V}_{\sigma_{n}} \in-\widehat{\mathcal{K}}_{\sigma_{n}, \sigma}
$$

for every $n \geq 1$. By letting $n$ tend to infinity, we obtain the cone constraint for $\Delta \widehat{V}_{\sigma}$.

By the Lebesgue density theorem, for a.e. $\omega$ and $d \operatorname{Var}_{t}\left(\widehat{V}^{c}\right)(\omega)$-a.e. $t \in[0, T]$, we have

$$
\begin{aligned}
\dot{\widehat{V}}_{t}^{c}(\omega) & =\lim _{\delta \downarrow 0} \frac{\widehat{V}_{t+\delta}^{c}(\omega)-\widehat{V}_{t}^{c}(\omega)}{\operatorname{Var}_{t+\delta}\left(\widehat{V}^{c}\right)(\omega)-\operatorname{Var}_{t}\left(\widehat{V}^{c}\right)(\omega)} \\
& \in \bigcap_{\delta>0}-\widehat{\mathcal{K}}_{t, t+\delta}(\omega) .
\end{aligned}
$$

Since $\left(\Pi_{t}\right)_{t \in[0, T]}$ is a càdlàg process, we have that $\cap_{\delta>0}-\widehat{\mathcal{K}}_{t, t+\delta}=-\widehat{K}_{t}$ a.s., so that $\dot{\widehat{V}}_{t}^{c}(\omega) \in-\widehat{K}_{t}(\omega)$, for a.e. $\omega$ and for $d \operatorname{Var}_{t}\left(\widehat{V}^{c}\right)(\omega)$-a.e. $t \in[0, T]$, which proves 1 .

To finish the proof of this lemma, let $\widehat{V}$ be a self-financing portfolio process with $\widehat{V}_{0}=$ 0 and let $Z \in \mathcal{Z}$. We now follow the arguments from [15], Lemma 3.1. Applying the integration by parts formula to the product $Z \widehat{V}^{c}$, we have

$$
\begin{aligned}
Z_{t} \widehat{V}_{t} & =Z_{t} \widehat{V}_{t}^{c}+Z_{t}\left(\sum_{u \leq t} \Delta \widehat{V}_{u}+\sum_{u<t} \Delta_{+} \widehat{V}_{u}\right) \\
& =\int_{0}^{t} \widehat{V}_{u}^{c} d Z_{u}+\int_{0}^{t} Z_{u} \dot{\hat{V}}_{u}^{c} d \operatorname{Var}_{u}\left(\widehat{V}^{c}\right)+Z_{t}\left(\sum_{u \leq t} \Delta \widehat{V}_{u}+\sum_{u<t} \Delta_{+} \widehat{V}_{u}\right) \\
& =\int_{0}^{t} \widehat{V}_{u} d Z_{u}+\int_{0}^{t} Z_{u} \dot{\hat{V}}_{u}^{c} d \operatorname{Var}_{u}\left(\widehat{V}^{c}\right)+\sum_{u \leq t} Z_{u-} \Delta \widehat{V}_{u}+\sum_{u<t} Z_{u} \Delta_{+} \widehat{V}_{u} .
\end{aligned}
$$

The first integral is clearly a local martingale as $\widehat{V}$ is locally bounded, which is a straightforward consequence of [8] (VIII.11, p. 337). The second one is a decreasing process, the 
product $Z_{t} \dot{\widehat{V}}_{t}^{c}$ being negative for a.e. $\omega$ and $d \operatorname{Var}_{t}\left(\widehat{V}^{c}\right)(\omega)$-a.e. $t \in[0, T]$. Moreover, the first and the second sum are both decreasing processes, since, for every predictable stopping time $\sigma, Z_{\sigma-} \Delta \widehat{V}_{\sigma} \leq 0$ a.s., and, for every stopping time $\tau, Z_{\tau} \Delta_{+} \widehat{V}_{\tau} \leq 0$ a.s.. Hence, $Z \widehat{V}$ is a local super-martingale for every $Z \in \mathcal{Z}$.

Assume now that $\widehat{V}$ is admissible with threshold $a>0$ and let $Z^{s} \in \mathcal{Z}^{s}$. In particular, we have that $Z_{\tau}^{s} \widehat{V}_{\tau} \geq-a \mathbf{1} Z_{\tau}^{s}$ a.s. for every stopping time $0 \leq \tau \leq T$. Finally, since $Z^{s} \widehat{V}$ is a local super-martingale bounded from below by the martingale $-a \mathbf{1} Z^{s}$, Fatou's lemma gives that $Z^{s} \widehat{V}$ is in fact a true super-martingale. The proof is now complete.

We conclude this section by comparing our definition of admissibility with that given in the paper [12] by Kabanov and Last, where the transaction costs are assumed to be constant. In fact, we will show in this case that the two definitions coincide. In order to do so, we briefly recall Kabanov's original framework, the definition of the transaction costs matrix $\Lambda$ and the related notion of admissibility.

In [12] (compare also to [11] and [15]) the bid-ask process $\left(\Pi_{t}\right)$ was introduced in the following way. These authors start with a $d$-dimensional price process $\left(S_{t}\right)_{t \in[0, T]}$ modelling the prices of $d$ assets without transaction costs in terms of some numéraire. One then defines a non-negative $d \times d$-matrix $\Lambda=\left(\lambda^{i j}\right)_{1 \leq i, j \leq d}$ of transaction cost coefficients, i.e., $\lambda^{i j} \geq 0$ models the proportional factor one has to pay in transaction costs, when exchanging the $i$ 'th for the $j$ 'th asset. Again it is natural to impose the condition that, for $1 \leq i, j, k \leq d$, we have

$$
\left(1+\lambda^{i j}\right) \leq\left(1+\lambda^{i k}\right)\left(1+\lambda^{k j}\right) .
$$

The transaction costs matrix $\Lambda$ may be constant (as in [12]), or may depend on $t$ and $\omega$ in an adapted way (as in [15]). In any case, letting

$$
\pi_{t}^{i j}(\omega)=\left(1+\lambda_{t}^{i j}(\omega)\right) \frac{S_{t}^{j}(\omega)}{S_{t}^{i}(\omega)}, \quad 1 \leq i, j \leq d,
$$

one obtains a bid-ask process $\left(\Pi_{t}\right)$. We refer to [17] for more detailed comments on how these two ways of modelling transaction costs are related.

For the rest of this section the transaction costs matrix $\Lambda$ is assumed to be constant (in $t$ and $\omega)$.

We recall the definitions of the solvency region (in terms of the numéraire in which the price process $S$ is denoted) $K$ and its dual $K^{*}$ as given, e.g., in [12]: let $M$ denote the set of all $x \in \mathbb{R}^{d}$ for which there exists a "transfer" matrix $\left(a^{i j}\right)_{1 \leq i, j \leq d}$ with non-negative entries such that

$$
x^{i}=-\sum_{j=1}^{d} a^{j i}+\sum_{j=1}^{d}\left(1+\lambda^{i j}\right) a^{i j}, \quad 1 \leq i \leq d .
$$

Then, $K:=M+\mathbb{R}_{+}^{d}$ and

$$
K^{*}=\left\{w \in \mathbb{R}_{+}^{d}: w^{j}-\left(1+\lambda^{i j}\right) w^{i} \leq 0,1 \leq i, j \leq d\right\} .
$$


Observe that

$$
\widehat{K}_{t}(\omega)=\left\{w \in \mathbb{R}^{d}:\left(w^{1} S_{t}^{1}(\omega), \ldots, w^{d} S_{t}^{d}(\omega)\right) \in K\right\}
$$

and

$$
\widehat{K}_{t}^{*}(\omega)=\left\{w \in \mathbb{R}^{d}:\left(w^{1} S_{t}^{1}(\omega), \ldots, w^{d} S_{t}^{d}(\omega)\right) \in K^{*}\right\} .
$$

Let now $\widehat{V}$ be a self-financing portfolio process as in Definition 7 . We will say that $\widehat{V}$ is admissible in Kabanov's sense if there exists a threshold $a>0$ such that $\widehat{V}_{\tau} \succeq_{\tau}-a \mathbf{1}$ a.s. for all stopping times $0 \leq \tau \leq T$.

We notice that in this context, i.e. $\Lambda$ constant, the efficient friction condition $\mathrm{EF}$ (Remark 6) translates into the property int $K^{*} \neq \emptyset$. In [12], instead of assuming SCPS, the authors assume EF and that there exists an equivalent martingale measure $\mathbf{Q}$ for the price process $S$, which is a stronger assumption than just SCPS. Furthermore, in [12] the price process $S$ is assumed to have continuous paths. In the next proposition, we drop the continuity assumption on $S$ and we show that, when the transaction costs matrix is constant, Kabanov's notion of admissibility and ours are equivalent.

Proposition 9 Assume that there exists a probability measure $\mathbf{Q} \sim \mathbf{P}$ such that $S$ is a Q-martingale. Let $\Lambda=\left(\lambda^{i j}\right)_{1 \leq i, j \leq d}$ be a constant transaction costs matrix such that the efficient friction condition int $K^{*} \neq \emptyset$ is satisfied and let $\widehat{V}$ be a self-financing portfolio process as in Definition 7. Then the assumption SCPS holds true and the following are equivalent:

(i) $\widehat{V}$ is admissible in the sense of Definition 7 above, i.e., there exists an a $>0$ such that $\widehat{V}_{T} \succeq_{T}-a \mathbf{1}$ and $Z_{\tau}^{s} \widehat{V}_{\tau} \geq-a Z_{\tau}^{s} \mathbf{1}$ a.s. for all stopping times $0 \leq \tau \leq T$ and for every $Z^{s} \in \mathcal{Z}^{s}$;

(ii) $\widehat{V}$ is admissible in Kabanov's sense, i.e. there exists an $a>0$ such that $\widehat{V}_{\tau} \succeq_{\tau}-a \mathbf{1}$ for all stopping times $0 \leq \tau \leq T$.

In fact, if one of the conditions is satisfied for some fixed $a>0$ the other one also holds true for the same threshold a.

Proof. We first show that, when $\Lambda$ is constant, EF plus the existence of an equivalent (true) martingale measure $\mathbf{Q}$ for $S$ imply SCPS. Indeed, fix an arbitrary $w \in \operatorname{int} K^{*}$ and consider the process $Z^{w}$ whose $i$ 'th component is defined by

$$
Z_{t}^{w, i}=w^{i} S_{t}^{i} D_{t} \quad 1 \leq i \leq d
$$

where $D_{t}:=\mathbf{E}\left[\frac{d \mathbf{Q}}{d \mathbf{P}} \mid \mathcal{F}_{t}\right]$. It is easily verified that $Z^{w}$ is a strictly consistent price process (compare [17]). Now we prove the equivalence between $(i)$ and (ii).

$(i i) \Rightarrow(i)$ Let $\widehat{V}$ and $Z^{s}$ be, respectively, a self-financing portfolio process and a strictly consistent price process. By $(i i)$, one has that $\widehat{V}_{\tau} \succeq_{\tau}-a \mathbf{1}$ for all stopping times $0 \leq \tau \leq T$, so that, using Remark 4, we have $Z_{\tau}^{s} \widehat{V}_{\tau} \geq-a Z_{\tau}^{s} \mathbf{1}$ for each stopping times $0 \leq \tau \leq T$. 
$(i) \Rightarrow(i i)$ Let $\widehat{V}$ be an admissible portfolio process as in Definition 7 with threshold $a>0$. This implies that $Z_{\tau}^{s}\left(\widehat{V}_{\tau}+a \mathbf{1}\right) \geq 0$ for all stopping times $0 \leq \tau \leq T$ and every $Z^{s} \in \mathcal{Z}^{s}$. Let $Z^{w}$ be a strictly consistent price process as in (4). Thus

$$
\sum_{i=1}^{d} w^{i}\left(S_{\tau}^{i} \widehat{V}_{\tau}^{i}+a S_{\tau}^{i}\right) \geq 0 \text { a.s., for all stopping times } 0 \leq \tau \leq T .
$$

Consider now a sequence $w_{n} \in \operatorname{int} K^{*}$ dense in $K^{*}$. From (5) we deduce that

$$
\mathbf{P}\left[\sum_{i=1}^{d} w_{n}^{i}\left(S_{\tau}^{i} \widehat{V}_{\tau}^{i}+a S_{\tau}^{i}\right) \geq 0, \forall n \geq 1\right]=1 .
$$

We can conclude that $S_{\tau}\left(\widehat{V}_{\tau}+a \mathbf{1}\right) \in K$ a.s. or, equivalently, $\widehat{V}_{\tau}+a \mathbf{1} \in \widehat{K}_{\tau}$ a.s. for all stopping times $0 \leq \tau \leq T$.

\section{The Closedness Property of the Cone of Attainable Claims}

Let us consider the set $\widehat{\mathcal{A}}_{T}^{x}$ of all contingent claims attainable with initial endowment $x \in \mathbb{R}^{d}$, which is defined as

$$
\widehat{\mathcal{A}}_{T}^{x}=\left\{\widehat{V}_{T}: \widehat{V} \in \widehat{\mathcal{V}}_{a d m}^{x}\right\} .
$$

Let us denote by $\widehat{\mathcal{V}}^{0, a}$, for $a>0$, the subset of all processes $\widehat{V} \in \widehat{\mathcal{V}}_{a d m}^{0}$ such that $\widehat{V}_{T} \succeq_{T}-a \mathbf{1}$.

In this section, we will show that the set $\widehat{\mathcal{A}}_{T}^{x}$ is Fatou-closed with respect to the order $\succeq_{T}$ on $L^{0}\left(\mathbb{R}^{d}, \mathcal{F}_{T}\right)$. By this we mean, in the present context, the following property: let $\widehat{V}_{T}^{n}$ be a sequence in $\widehat{\mathcal{A}}_{T}^{x}$ converging a.s. to a r.v. $X \in L^{0}\left(\mathbb{R}^{d}, \mathcal{F}_{T}\right)$ and such that $\widehat{V}^{n} \in \widehat{\mathcal{V}}^{0, a}$ for all $n \geq 1$ and for a given $a>0$. Then there exists a portfolio process $\widehat{V}^{0} \in \widehat{\mathcal{V}}_{a d m}^{x}$ with terminal value $X$.

Once this property is proved, it will be fairly standard to establish a super-replication theorem with proportional transaction costs, by using essentially the same arguments as in [15].

At this point, we need some preparatory results. The idea underlying the next two lemmas goes back to [15], Lemma 3.3, where Kabanov and Stricker proved a similar bound, but using a different notion of portfolio process.

The following definition will be useful in the next lemma: given a convex cone $C \subseteq \mathbb{R}^{d}$ and a real number $\epsilon>0$, we define the $\epsilon$-interior of $C$ as the set of all $x \in C$ such that $B\left(x, \epsilon\|x\|_{d}\right) \subseteq C$, where $B\left(x, \epsilon\|x\|_{d}\right)$ is the open ball centered at $x$ and with radius $\epsilon\|x\|_{d}$. Clearly this defines a convex cone which will be denoted by $\epsilon$-int $C$. Let $C^{*}$ denote the polar of $C$. The following elementary fact will be helpful in the sequel:

$$
x \in \epsilon \text {-int } C \text { if and only if } x y \geq \epsilon\|x\|_{d} \text { for every } y \in C^{*} \text { with }\|y\|_{d}=1 .
$$

Furthermore, let $Z^{s}$ be a strictly consistent price process. We denote by $\varepsilon$ the biggest random variable (in the essential supremum sense) belonging to $L^{0}\left(\mathbb{R}_{+}, \mathcal{F}_{T}\right)$ such that $Z_{t}^{s} \in$ $\varepsilon$-int $\widehat{K}_{t}^{*}$ for all $t \in[0, T]$ a.s., i.e.

$$
\varepsilon:=\operatorname{ess} \sup \left\{\eta \in L^{0}\left(\mathbb{R}_{+}, \mathcal{F}_{T}\right): Z_{t}^{s} \in \eta \text {-int } \widehat{K}_{t}^{*}, \forall t \in[0, T] \text { a.s. }\right\} .
$$


Lemma 10 Let $Z^{s}$ be a strictly consistent price process for the bid-ask process $\left(\Pi_{t}\right)_{t \in[0, T]}$. Then,

$$
\mathbf{P}[\varepsilon>0]=1,
$$

where $\varepsilon$ is defined as in (8). As a consequence, $\mathbf{P}\left[Z_{t}^{s} \in \varepsilon\right.$-int $\left.\widehat{K}_{t}^{*}, \forall t \in[0, T]\right]=1$.

Proof. Consider the following increasing sequence of $[0, T] \cup\{\infty\}$-valued stopping times

$$
\tau_{n}:=\inf \left\{t: Z_{t}^{s} \notin \frac{1}{n} \text {-int } \widehat{K}_{t}^{*}\right\}, \quad n \geq 1,
$$

with the usual convention $\inf \emptyset=\infty$, and set $\tau:=\sup _{n \geq 1} \tau_{n}$. As $Z^{s}$ is a strictly consistent price process, $\tau$ is a predictable $[0, T] \cup\{\infty\}$-valued stopping time. Indeed, the sequence $\tau_{n}$ announces $\tau$ on the set $\{\tau<\infty\}$.

To prove the statement it suffices to show that $\mathbf{P}[\tau<\infty]=0$. To do so, notice that on $\{\tau<\infty\}$ we have $\tau_{n}<\infty$ and so $Z_{\tau_{n}}^{s} \notin \frac{1}{n}$-int $\widehat{K}_{\tau_{n}}^{*}$ for all $n \geq 1$. Being $Z^{s}$ càdlàg, $Z_{\tau_{n}}^{s}$ tends a.s. to $Z_{\tau-}^{s}$ on $\{\tau<\infty\}$. Since also $\left(\widehat{K}_{t}^{*}\right)_{t \in[0, T]}$ is a càdlàg process, $Z_{\tau-}^{s}$ must lie at the boundary $\partial \widehat{K}_{\tau-}^{*}$ of $\widehat{K}_{\tau-}^{*}$ a.s. on $\{\tau<\infty\}$. Furthermore, $Z_{\tau-}^{s}$ is a.s. different from zero on $\{\tau<\infty\}$. This follows from the fact that, since $Z^{s}$ is an $\mathbb{R}_{+}^{d} \backslash\{0\}$-valued martingale, one has $\inf _{t \in[0, T]}\left\|Z_{t}^{s}\right\|_{d}>0$ a.s.. Hence we have that $Z_{\tau-}^{s} \in \partial \widehat{K}_{\tau-}^{*} \backslash\{0\}$ a.s. on $\{\tau<\infty\}$, which of course contradicts the fact that $Z_{\tau-}^{s}$, being a strictly consistent price process, must belong to int $\widehat{K}_{\tau-}^{*}$ a.s. on $\{\tau<\infty\}$. Hence $\mathbf{P}[\tau<\infty]=0$.

In particular, we have $Z_{t}^{s} \in \varepsilon$-int $\widehat{K}_{t}^{*}$ a.s. for all $t \in[0, T]$. Since both processes $\left(Z_{t}^{s}\right)_{t \in[0, T]}$ and $\left(\widehat{K}_{t}^{*}\right)_{t \in[0, T]}$ are càdlàg, we can conclude.

The following lemma provides a uniform bound for the total variation of any family of admissible portfolio processes with the same threshold $a>0$. We recall that $\widehat{\mathcal{V}}^{0, a}$ denotes the subset of all admissible portfolio processes (starting from 0) $\widehat{V} \in \widehat{\mathcal{V}}_{a d m}^{0}$ such that $\widehat{V}_{T} \succeq_{T}-a \mathbf{1}$.

Lemma 11 Suppose that $\left(\Pi_{t}\right)_{t \in[0, T]}$ is such that SCPS holds. Then, there exists a probability $\mathbf{Q} \sim \mathbf{P}$ and a constant $C>0$ such that, for $a>0$ and $\widehat{V} \in \widehat{\mathcal{V}}^{0, a}$,

$$
\mathbf{E}_{\mathbf{Q}}\left[\operatorname{Var}_{T}(\widehat{V})\right] \leq C \cdot a .
$$

Proof. Let $Z^{s}$ be a strictly consistent price process. For each admissible portfolio process $\widehat{V}$ with admissibility threshold $a$, the super-martingale property of the process $Z^{s} \widehat{V}$ stated in Lemma 8 together with a standard application of Fatou's lemma ensures that

$$
\begin{aligned}
\mathbf{E}\left[-\int_{0}^{T} Z_{u}^{s} \hat{\bar{V}}_{u}^{c} d \operatorname{Var}_{u}\left(\widehat{V}^{c}\right)-\sum_{u \leq T} Z_{u}^{s} \Delta \widehat{V}_{u}-\sum_{u \leq T} Z_{u}^{s} \Delta_{+} \widehat{V}_{u}\right] & \leq-\mathbf{E}\left[Z_{T}^{s} \widehat{V}_{T}\right] \\
& \leq a \mathbf{E}\left[Z_{T}^{s} \mathbf{1}\right]<\infty .
\end{aligned}
$$

Notice that, since $Z^{s}$ is an $\mathbb{R}_{+}^{d} \backslash\{0\}$-valued martingale, we have $\inf _{t \in[0, T]}\left\|Z_{t}^{s}\right\|_{d}>0$ a.s.. Set $\alpha:=\varepsilon \inf _{t \in[0, T]}\left\|Z_{t}^{s}\right\|_{d}$ where $\varepsilon$ is defined as in (8). Lemma 10 together with (7) implies that

$$
\mathbf{P}\left[Z_{t}^{s} \widehat{V}_{t} \geq \alpha>0, \forall t \in[0, T]\right]=1
$$


Then the left-hand side of inequality (11) is bigger than

$$
\mathbf{E}\left[\alpha\left(\operatorname{Var}_{T}\left(\widehat{V}^{c}\right)+\sum_{u \leq T}\left\|\Delta \widehat{V}_{u}\right\|_{d}+\sum_{u \leq T}\left\|\Delta_{+} \widehat{V}_{u}\right\|_{d}\right)\right]
$$

which yields that

$$
\sup _{\widehat{V} \in \widehat{\mathcal{V}}^{0, a}} \mathbf{E}\left[\alpha\left(\operatorname{Var}_{T}\left(\widehat{V}^{c}\right)+\sum_{u \leq T}\left\|\Delta \widehat{V}_{u}\right\|_{d}+\sum_{u \leq T}\left\|\Delta_{+} \widehat{V}_{u}\right\|_{d}\right)\right] \leq a \mathbf{E}\left[Z_{T}^{s} \mathbf{1}\right] .
$$

Since

$$
\operatorname{Var}_{T}(\widehat{V})=\operatorname{Var}_{T}\left(\widehat{V}^{c}\right)+\sum_{u \leq T}\left\|\Delta \widehat{V}_{u}\right\|_{d}+\sum_{u \leq T}\left\|\Delta_{+} \widehat{V}_{u}\right\|_{d}
$$

setting $g:=\mathbf{E}\left[Z_{T}^{s} \mathbf{1}\right]^{-1} \alpha, C:=\mathbf{E}[g]$ and $\frac{d \mathbf{Q}}{d \mathbf{P}}:=\frac{g}{C}$ concludes the proof.

In order to show that the set $\widehat{\mathcal{A}}_{T}^{0}$ is Fatou-closed we will use the following Proposition 13, which is a compactness result for predictable finite variation processes. To prove this result we will repeatedly use an easy consequence of a "Komlós-like" result proved by Delbaen and Schachermayer [6] (Proposition A1.1, p. 515), that we recall for the reader's convenience.

Proposition 12 Given a sequence of real-valued functions $f_{n}$ bounded in $\in L^{1}(\mathbf{Q})$, there are convex combinations

$$
g_{n} \in \operatorname{conv}\left(f_{n}, f_{n+1}, \ldots\right)
$$

such that $g_{n}$ converges a.s. to some $g_{0} \in L^{1}(\mathbf{Q})$.

We now state the parametrized version of Helly's theorem which will be the central tool for proving Theorem 14 below.

Proposition 13 Let $V^{n}$ be a sequence of finite variation, predictable processes such that the corresponding sequence $\operatorname{Var}_{T}\left(V^{n}\right)$ is bounded in $L^{1}(\mathbf{Q})=L^{1}\left(\mathcal{F}_{T}, \mathbf{Q}\right)$ for some probability $\mathbf{Q} \sim \mathbf{P}$. Then, there exists a sequence $W^{n} \in \operatorname{conv}\left\{V_{n}, V_{n+1}, \ldots\right\}$ such that $W^{n}$ converges for a.e. $\omega$ for every $t \in[0, T]$ to a finite variation, predictable process $W^{0}$, i.e.

$$
\mathbf{P}\left[W_{t}^{n} \rightarrow W_{t}^{0}, \forall t \in[0, T]\right]=1 .
$$

Proof. Let $V^{n}$ be a sequence as in the statement and set $D:=([0, T] \cap \mathbb{Q}) \cup\{T\}$. By assumption, the sequence $\operatorname{Var}_{T}\left(V^{n}\right)$ is bounded in $L^{1}(\mathbf{Q})$, for some probability $\mathbf{Q} \sim \mathbf{P}$, so that we can use Proposition 12 together with a diagonalization procedure to obtain sequences of convex weights $\alpha_{n}^{j}$ such that for

$$
\mathbb{W}_{t}^{n}=\sum_{j \geq 1} \alpha_{n}^{j} \operatorname{Var}_{t}\left(V^{n+j}\right) \in \operatorname{conv}\left(\operatorname{Var}_{t}\left(V^{n}\right), \operatorname{Var}_{t}\left(V^{n+1}\right), \ldots\right), \quad t \in D,
$$

and

$$
W_{t}^{n}=\sum_{j \geq 1} \alpha_{n}^{j} V_{t}^{n+j} \in \operatorname{conv}\left(V_{t}^{n}, V_{t}^{n+1}, \ldots\right), \quad t \in D
$$


there exist $\mathcal{F}_{t}$-measurable random variables $\widetilde{\mathbb{W}}_{t}^{0}$ and $W_{t}^{0}$ which are, respectively, real-valued and $\mathbb{R}^{d}$-valued, such that

$$
\mathbb{W}_{t}^{n} \rightarrow \widetilde{\mathbb{W}}_{t}^{0}, \quad t \in D
$$

and

$$
W_{t}^{n} \rightarrow W_{t}^{0}, \quad t \in D,
$$

almost surely. We denote by $\widetilde{\Omega}_{0}$ the event where both (13) and (14) hold true so that $\mathbf{P}\left[\widetilde{\Omega}_{0}\right]=1$.

Observe now that $q \mapsto \widetilde{\mathbb{W}}_{q}^{0}(\omega)$ is increasing over $D$ for all $\omega \in \widetilde{\Omega}_{0}$, so that we can set

$$
\mathbb{W}_{t}^{0}=\lim _{q \downarrow \downarrow t, q \in \mathbb{Q}} \widetilde{\mathbb{W}}_{q}^{0}, \quad t \in[0, T)
$$

and $\mathbb{W}_{T}^{0}=\widetilde{\mathbb{W}}_{T}^{0}$ on $\widetilde{\Omega}_{0}$.

Claim: let $\left.(\omega, t) \in \widetilde{\Omega}_{0} \times\right] 0, T[$ be such that $t$ is a continuity point of the function $s \mapsto \mathbb{W}_{s}^{0}(\omega)$. Then $W_{t}^{n}(\omega)$ converges in $\mathbb{R}^{d}$.

Indeed, for $\epsilon>0$ let $q_{1}<t<q_{2}$ be rational numbers such that $\mathbb{W}_{q_{2}}^{0}(\omega)-\mathbb{W}_{q_{1}}^{0}(\omega)<\epsilon$. Choose rationals $r_{1}, r_{2}$ such that $q_{1}<r_{1}<t<r_{2}<t_{2}$. Then there is $N \in \mathbb{N}$ such that, for $n \geq N$, we have $\mathbb{W}_{r_{2}}^{n}(\omega)-\mathbb{W}_{r_{1}}^{n}(\omega)<2 \epsilon$. Using the inequality

$$
\left\|W_{t}^{n}(\omega)-W_{r_{1}}^{n}(\omega)\right\| \leq \mathbb{W}_{r_{2}}^{n}(\omega)-\mathbb{W}_{r_{1}}^{n}(\omega)<2 \epsilon
$$

we conclude that the convergence of $W_{q}^{n}(\omega)$ on the rationals $\mathbb{Q} \cap[0, T]$ implies the convergence of $W_{t}^{n}(\omega)$ on all points of continuity of $s \mapsto \mathbb{W}_{s}^{0}(\omega)$. We denote by $W_{t}^{0}(\omega)$ the limit of the sequence $W_{t}^{n}(\omega)$ on these points of continuity $(\omega, t)$.

To conclude the proof we still have to assure the convergence of $W_{t}^{n}(\omega)$ at the points of discontinuity of $s \mapsto \mathbb{W}_{s}^{0}(\omega)$, for a set $\Omega_{0}$ of full $\mathbf{P}$-measure. We may proceed as follows: being $\mathbb{W}^{0}$ an increasing, right-continuous adapted process over $[0, T]$, we can apply Théorème 117 [7], ensuring that there exists a sequence $\tau_{k}$ of $[0, T] \cup\{\infty\}$-valued stopping times exhausting the jump instants of the process $\mathbb{W}^{0}$, i.e. $\left\{\Delta \mathbb{W}^{0} \neq 0\right\} \subseteq \cup_{k \geq 1} \llbracket \tau_{k} \rrbracket$. We set $J:=\cup_{k \geq 1} \llbracket \tau_{k} \rrbracket$. By taking once more convex combinations, we can assume w.l.o.g. that,

$$
W_{\tau_{k}}^{n} \rightarrow W_{\tau_{k}}^{0} \quad \text { a.s. on }\left\{\tau_{k} \leq T\right\},
$$

for every $k$. We set $\Omega_{0}:=\left\{\omega \in \widetilde{\Omega}_{0}:(15)\right.$ holds true $\}$ so that we still have $\mathbf{P}\left[\Omega_{0}\right]=1$.

As a consequence, for $\omega \in \Omega_{0}, W_{t}^{n}(\omega) \rightarrow W_{t}^{0}(\omega)$ for every $t \in[0, T]$. Clearly, the limit $W^{0}$ is a finite variation, predictable process. This concludes the proof.

Now, we state and prove the main result of this paper.

Theorem 14 Let $\left(\Pi_{t}\right)_{t \in[0, T]}$ be a bid-ask process such that SCPS holds true. Then, the set $\widehat{\mathcal{A}}_{T}^{0}$ is Fatou-closed. 
Proof. Let $\widehat{V}^{n}$ be a sequence in $\widehat{\mathcal{V}}^{0, a}$ for some $a>0$, and such that $\widehat{V}_{T}^{n}$ tends a.s. to some $X \in L^{0}\left(\mathcal{F}_{T}, \mathbb{R}^{d}\right)$. By Lemma 11 and Proposition 13, there exists a sequence of convex combinations of $\widehat{V}^{n}$, still denoted by $\widehat{V}^{n}$, which converges to some finite variation, predictable process $\widehat{V}^{0}$ in the following sense:

$$
\mathbf{P}\left[\widehat{V}_{t}^{n} \rightarrow \widehat{V}_{t}^{0}, \forall t \in[0, T]\right]=1 .
$$

It is now clear that the limiting process $\widehat{V}^{0}$ belongs to $\widehat{\mathcal{V}}_{a d m}^{x}$ and $\widehat{V}_{T}^{0}=X$ a.s.. Indeed, it trivially satisfies the cone constraints (2) so that it is a self-financing portfolio process. Furthermore, since each $\widehat{V}^{n}$ is an admissible portfolio process with threshold $a$, passing to the limit gives $Z_{\tau}^{s} \widehat{V}_{\tau}^{0} \geq-a Z_{\tau}^{s} \mathbf{1}$ a.s. for all stopping times $0 \leq \tau \leq T$ and every $Z^{s} \in \mathcal{Z}^{s}$. Hence $\widehat{V}^{0}$ is an admissible portfolio process (with threshold $a$ ).

\section{The Super-Replication Theorem}

Let $X \in L^{0}\left(\mathbb{R}^{d}, \mathcal{F}_{T}\right)$ be a vector-valued contingent claim such that $X \succeq_{T}-a \mathbf{1}$ for some constant $a>0$. Let us consider the following sets:

- $\Gamma(X):=\left\{x \in \mathbb{R}^{d}: X \in \widehat{\mathcal{A}}_{T}^{x}\right\}$,

- $\mathbb{D}(X):=\left\{x \in \mathbb{R}^{d}: Z_{0} x \geq \mathbf{E}\left[Z_{T} X\right], \forall Z \in \mathcal{Z}\right\}$,

- $\mathbb{D}^{s}(X):=\left\{x \in \mathbb{R}^{d}: Z_{0} x \geq \mathbf{E}\left[Z_{T} X\right], \forall Z \in \mathcal{Z}^{s}\right\}$.

In the sequel, $\chi_{A}$ will denote the indicator function of the set $A$.

Theorem 15 Let $X \in L^{0}\left(\mathbb{R}^{d}, \mathcal{F}_{T}\right)$ be such that $X \succeq_{T}-a \mathbf{1}$ for some $a>0$. Under Assumption 5 (SCPS), we have

$$
\Gamma(X)=\mathbb{D}(X)=\mathbb{D}^{s}(X) .
$$

Proof. We proceed as in Kabanov and Stricker [15], Lemmas 3.6 and 3.7 with some minor modifications.

1. $\Gamma(X) \subseteq \mathbb{D}(X)$ : this is an immediate consequence of the super-martingale property of portfolio processes stated in Lemma 8.

2. $\mathbb{D}(X) \subseteq \mathbb{D}^{s}(X)$ : this is trivially satisfied.

3. $\mathbb{D}^{s}(X) \subseteq \Gamma(X)$. In order to apply Theorem 3.9 in [15], let us first show that the set $\widehat{\mathcal{A}}_{T}^{x} \cap L^{\infty}\left(\mathbb{R}^{d}, \mathcal{F}_{T}\right)$ is Fatou-dense in $\widehat{\mathcal{A}}_{T}^{x}$ : that is, for every $\widehat{V}_{T} \in \widehat{\mathcal{A}}_{T}^{x}$ there exists a sequence $\widehat{V}_{T}^{n} \in \widehat{\mathcal{A}}_{T}^{x} \cap L^{\infty}\left(\mathbb{R}^{d}, \mathcal{F}_{T}\right)$ such that $\widehat{V}_{T}^{n} \rightarrow \widehat{V}_{T}$ a.s. and $\widehat{V}_{T}^{n} \succeq_{T}-a \mathbf{1}$, where $a>0$ is the threshold given by Definition 7. Fix $\widehat{V}_{T}$ and let us define the sequence $\widehat{V}_{T}^{n}$ as follows:

$$
\widehat{V}_{T}^{n}:=\widehat{V}_{T} \chi_{\left\{\left|\widehat{V}_{T}\right| \leq n\right\}}-a \chi_{\left\{\left|\widehat{V}_{T}\right|>n\right\}} .
$$

Clearly $\widehat{V}_{T}^{n} \in \widehat{\mathcal{A}}_{T}^{x} \cap L^{\infty}\left(\mathbb{R}^{d}, \mathcal{F}_{T}\right)$. Furthermore, we also have that

$$
\widehat{V}_{T}-L^{\infty}\left(\widehat{K}_{T}, \mathcal{F}_{T}\right) \subseteq \widehat{\mathcal{A}}_{T}^{x} \cap L^{\infty}\left(\mathbb{R}^{d}, \mathcal{F}_{T}\right)
$$


for all $\widehat{V}_{T} \in \widehat{\mathcal{A}}_{T}^{x} \cap L^{\infty}\left(\mathbb{R}^{d}, \mathcal{F}_{T}\right)$.

Theorem 3.9 in [15] now applies, yielding

$$
\widehat{\mathcal{A}}_{T}^{x}=\left\{Y \in L_{b}^{0}\left(\mathbb{R}^{d}, \mathcal{F}_{T}\right): \mathbf{E}[\eta Y] \leq \sup _{\widehat{V}_{T} \in \widehat{\mathcal{A}}_{T}^{x}} \mathbf{E}\left[\eta \widehat{V}_{T}\right], \forall \eta \in L^{1}\left(\widehat{K}_{T}^{*}, \mathcal{F}_{T}\right)\right\},
$$

where $L_{b}^{0}\left(\mathbb{R}^{d}, \mathcal{F}_{T}\right)$ is the set of all $\mathbb{R}^{d}$-valued and $\mathcal{F}_{T}$-measurable random variables $Y$ such that $Y \succeq_{T}-a \mathbf{1}$, for some $a>0$. For $x \notin \Gamma(X)$ we have $X \notin \widehat{\mathcal{A}}_{T}^{x}$. Hence (17) implies that there exists $\eta \in L^{1}\left(\widehat{K}_{T}^{*}, \mathcal{F}_{T}\right)$ such that

$$
\mathbf{E}[\eta X]>\sup _{\widehat{V}_{T} \in \widehat{\mathcal{A}}_{T}^{x}} \mathbf{E}\left[\eta \widehat{V}_{T}\right] .
$$

Let us define the process $Z_{t}:=\mathbf{E}\left[\eta \mid \mathcal{F}_{t}\right], t \in[0, T]$. By definition, $\mathbf{E}\left[Z_{T} X\right]>Z_{0} x$. Moreover $Z$ is a consistent price process. Indeed, let us consider the following process:

$$
\widehat{W}_{u}=x-\vartheta \zeta \chi_{] t, T]}(u), \quad u \in[0, T],
$$

for some $t \in[0, T]$ and arbitrary random variables $\vartheta \in L^{\infty}\left(\mathbb{R}_{+}, \mathcal{F}_{t}\right)$ and $\zeta \in L^{\infty}\left(\widehat{K}_{t}, \mathcal{F}_{t}\right)$. Being a.s. bounded, $\widehat{W}$ belongs to $\widehat{\mathcal{V}}_{a d m}^{x}$, and

$$
\mathbf{E}\left[\eta \widehat{W}_{T}\right]=\mathbf{E}\left[Z_{T} \widehat{W}_{T}\right]=\mathbf{E}\left[Z_{T} \widehat{W}_{t+}\right]=\mathbf{E}\left[Z_{t} \widehat{W}_{t+}\right]=-\mathbf{E}\left[\vartheta Z_{t} \zeta\right],
$$

where the third equality comes from the fact that $\widehat{W}_{t+}$ is $\mathcal{F}_{t}$-measurable. Hence, (18) implies that $\mathbf{E}\left[\vartheta Z_{t} \zeta\right]>-\mathbf{E}[\eta X]$ and, $\vartheta$ being arbitrary, we can easily deduce that $Z_{t} \zeta \geq 0$ for all $\zeta \in L^{\infty}\left(\widehat{K}_{t}, \mathcal{F}_{t}\right)$, yielding $Z_{t} \in \widehat{K}_{t}^{*}$ a.s..

To finish the proof, it remains to modify $Z$ in order to have a strictly consistent price process $Z^{s}$ such that $\mathbf{E}\left[Z_{T}^{s} X\right]>Z_{0}^{s} x$. Proceeding as in [17], let $Z^{s}$ be a strictly consistent price process (whose existence is granted by definition). For $0<\beta \leq 1$ sufficiently small, the process $Z_{t}^{\beta}:=\beta Z_{t}^{s}+Z_{t}$ is a strictly consistent price process and we still have $\mathbf{E}\left[Z_{T}^{\beta} X\right]>Z_{0}^{\beta} x$. The proof is now complete.

Remark 16 Observe that Assumption 2 does not restrict the generality of our setting. All our arguments apply to the general case, where possibly $\mathcal{F}_{T-} \varsubsetneqq \mathcal{F}_{T}$ and $\Pi_{T-} \neq \Pi_{T}$. In fact, in order to do so, it suffices to enlarge the time interval $[0, T]$ to $[0, T+1]$ and assume that, between $T$ and $T+1$, the underlying filtration as well as the bid-ask process do not change, i.e., for every $t \in[T, T+1], \mathcal{F}_{t}=\mathcal{F}_{T}$ and $\Pi_{t}=\Pi_{T}$. Between $T$ and $T+1$, an agent is allowed to make a final self-financing change $\widehat{V}_{T+1}-\widehat{V}_{T} \in-\widehat{K}_{T}$ in her portfolio $\left(\widehat{V}_{t}\right)_{t \in[0, T+1]}$, according to the terms fixed by the market at time $T$. With these modifications, it is rather obvious that our version of the super-replication theorem applies also to contingent claims which are $\mathcal{F}_{T}$-measurable and not necessarily $\mathcal{F}_{T-\text {-measurable. }}$

In the same spirit we remark that in Definition 7 the final condition could be equivalently reformulated in a somewhat more elegant way: we may modify the property

(i) there is a constant $a>0$ such that $\widehat{V}_{T} \succeq_{T}-a \mathbf{1}$ and $Z_{\tau}^{s} \widehat{V}_{\tau} \geq-a Z_{\tau}^{s} \mathbf{1}$ a.s. for all stopping times $0 \leq \tau \leq T$ and for every $Z^{s} \in \mathcal{Z}^{s}$, 
by simply dropping the first requirement, i.e.

(ii) there is a constant $a>0$ such that $Z_{\tau}^{s} \widehat{V}_{\tau} \geq-a Z_{\tau}^{s} \mathbf{1}$ a.s. for all stopping times $0 \leq \tau \leq T$ and for every $Z^{s} \in \mathcal{Z}^{s}$.

Indeed, consider the model with the new terminal date $T+1$ as described above and set

$$
\widehat{\mathbb{K}}_{T+1}:=\left\{Y \in L^{0}\left(\mathcal{F}_{T}, \mathbb{R}^{d}\right): Z_{T+1}^{s} Y \geq 0 \text { a.s. } \forall Z^{s} \in \mathcal{Z}^{s}\right\},
$$

where we put $Z_{T+1}^{s}=Z_{T}^{s}, Z^{s} \in \mathcal{Z}^{s}$. Now, define the order $\succeq_{T+1}$ in terms of the closed

convex cone $\widehat{\mathbb{K}}_{T+1}$ in the usual way. It is clear that for this time-extended market conditions (i) and (ii) are equivalent and that the whole theory can be carried over with no changes. Although formulation (ii) seems slightly more elegant to us we have chosen the version (i) in Definition 7 above, mainly in order to continue in the spirit of the previous literature (e.g., [12], [15]).

Remark 17 An inspection of the above arguments reveals also that all our results can be extended to the case where $\left(\widehat{K}_{t}\right)_{t \in[0, T]}$ is just a càdlàg process with values in the closed polyhedral proper cones of $\mathbb{R}^{d}$ containing $\mathbb{R}_{+}^{d}$, where "proper" means that the cone does not contain a non-trivial subspace of $\mathbb{R}^{d}$ and the notion of convergence is defined in a rather obvious way.

\section{References}

[1] Ansel, J.P., and Stricker, Ch. (1994): Couverture des actifs contingents et prix maximum. Annales de l'Institut H. Poincaré, 30, 303-315.

[2] Bouchard, B. and Touzi, N. (2000): Explicit solution of the multivariate superreplication problem under transaction costs. Annals of Applied Probability, 10, 685708.

[3] Cvitanić, J. and Karatzas, I. (1996): Hedging and portfolio optimization under transaction costs: a martingale approach. Mathematical Finance, 6 (2), 133-165.

[4] Cvitanić, J., Pham, H. and Touzi, N. (1999): A closed form solution to the problem of super-replication under transaction costs. Finance and Stochastics, 3, 35-54.

[5] Delbaen, F., Kabanov, Yu.M., Valkeila, S. (2002): Hedging under transaction costs in currency markets: a discrete-time model. Mathematical Finance, 12 (1), 45-61.

[6] Delbaen, F. and Schachermayer, W. (1994): A general version of the fundamental theorem of asset pricing. Mathematische Annalen, 300, 463-520.

[7] Dellacherie, C. and Meyer, P.-A. (1975). Probabilités et potentiel, Chapitres I à IV. Hermann, Paris. 
[8] Dellacherie, C. and Meyer, P.-A. (1980). Probabilités et potentiel, Chapitres V à VIII. Hermann, Paris.

[9] El Karoui, N., and Quenez, M.-C. (1995): Dynamic Programming and Pricing of Contingent Claims in an Incomplete Market. SIAM Journal of Control and Optimization, 33 (1), 27-66.

[10] He, S., Wang, J., Yan, J. (1992): Semimartingale Theory and Stochastic Calculus. Science Press and CRC Press Inc., Beijing/Boca Raton.

[11] Kabanov, Yu.M. (1999): Hedging and liquidation under transaction costs in currency markets. Finance and Stochastics, 3 (2), 237-248.

[12] Kabanov, Yu.M. and Last, G. (2002): Hedging under transaction costs in currency markets: a continuous-time model. Mathematical Finance, 12 (1), 63-70.

[13] Kabanov, Yu.M., Rásonyi M. and Stricker, Ch. (2002): No-arbitrage criteria for financial markets with efficient friction. Finance and Stochastics, 6 (3), 371-382.

[14] Kabanov, Yu.M., Rásonyi M. and Stricker, Ch. (2003): On the closedness of sums of convex cones in $L^{0}$ and the robust no-arbitrage property. Finance and Stochastics, 6 (3), 403-411.

[15] Kabanov, Yu.M. and Stricker, Ch. (2002): Hedging of contingent claims under transaction costs. In: Sandman, K., Schönbucher, Ph. (eds) Advances in Finance and Stochastics. Essays in Honour of Dieter Sondermann. Berlin Heidelberg New York: Springer.

[16] Rásonyi, M. (2003): A remark on the superhedging theorem under transaction costs. Séminaires de Probabilités XXXVII. Lecture Notes in Mathematics 1832, 394-398. Springer Verlag.

[17] Schachermayer, W. (2004): The Fundamental Theorem of Asset Pricing under Proportional Transaction Costs in Finite Discrete Time. Mathematical Finance, 14 (1), $19-48$.

[18] Sin, C.A. (1996): Strictly local martingales and hedge ratios on stochastic volatility models. PhD-dissertation, Cornell University.

[19] Soner, H.M., Shreve, S.E. and Cvitanić, J. (1995): There is nontrivial hedging portfolio for option pricing with transaction costs. The Annals of Applied Probability, 5, 327-355.

[20] Yan, J.-A. (1998): A new look at the fundamental theorem of asset pricing. J. Korean Math. Soc., 35 (3), 659-673. 